\title{
Adaptive Policy Responses to Climate Change Scenarios in the Musi Catchment, India
}

\author{
Brian Davidson $^{1}$, Biju George ${ }^{2}$, Hector Malano ${ }^{1}$, and Petra Hellegers ${ }^{3}$ \\ ${ }^{1}$ University of Melbourne, Parkville \\ Victoria 3010, Australia \\ ${ }^{2}$ Integrated Water and Land Management Program, ICARDA, P.O. Box 2416 \\ Cairo, Egypt \\ ${ }^{3}$ Wageningen UR, P.O. Box 47, 6700 AA \\ Wageningen, the Netherlands
}

\begin{abstract}
In India the stresses on water resource systems have increased, due in part to increased demand for scarce water supplies. Yet, what could be of greater concern is the potential long-run threats of climate change affecting supplies. Before thinking of a policy response to these long-run concerns, the impact of climate change on the reliability of water supply across a catchment needs to be gauged from both a physical and an economic perspective. Once these impacts are known, a more target approach to policy can be formulated. The aim in this research is to briefly present and comment on the results of an assessment of some these dynamic interacting forces across the Musi catchment in India. Of primary interest are the impacts of three different climate variants over the next 30 years have on the reliability of water supply (at the 70, 80 and 90\% levels) across seven different agricultural zones in the Musi catchment in India. A hydro-economic modelling effort underlies these results (see Davidson et al forthcoming) which draws on a hydrologic analysis based on the Hadley climate model to model the surface and ground water in the catchment. This model then provides inputs into an allocation model (REALM), to assess the amount of water reliably supplied to different zones in the Musi catchment at different levels of reliability. These flows are ultimately valued to determine the economic consequences of different climate scenarios. In this study, the results are reported for four dryland regions (zones 1 to 4), an irrigation region (Musi Medium,) and two river diverters (the Musi Anicut and the Wastewater irrigation system). Unsurprisingly, from a physical perspective the region's most greatly affected are those heavily dependent on dryland agriculture, especially near Hyderabad City. This region is where the high value products (such as vegetables) are produced. These results imply that some caution should be exercised in choosing policies that allow for the adaptation of climate change, especially in the dryland zones.
\end{abstract}

\section{Introduction}

Policy makers in the water resource sector face multiple difficulties when confronted with developing long term strategies designed to adapt to the problems of dealing with climate change. A major constraint faced by policy makers is that they usually have limited resources to commit to the problem and as such they need to choose where to place these resources within the catchment. Catchments, while being contiguous, are extremely complex spatially and temporally diverse natural systems that are used for multiple uses. So, with limited resources should the policy makers direct their attention in adaptation to where most of the water goes, where it is most valuably used, or any number of other considerations that attract consideration.

The first questions that a policy maker would ask in such a situation is: What are the physical impacts from climate change likely to be, especially regarding the impacts on precipitation? That, in itself, is a challenging proposition because those responsible for forecasting climate change usually put out an ensemble of results; some which end up with precipitation rising and others with it falling. And where is the impact going to be felt most? In an attempt to get a more definitive answer to the question of what is the more likely climate, most of the research focuses on alternative carbon mitigation strategies, how carbon emissions reduction policies are working, etc. This research focus is rather meaningless to the policy-maker because they are out of their sphere of influence to put an adaptation policy in place in a particular catchment or river basin.

One approach to resolving this problem (the one followed in this paper), is to look at an ensemble of scenarios modelled and take an average of the outcomes. Then measure the differences between an average, a wetter and a dryer 
climate outcome and compare them. Two elements should be looked for in this comparison. First, are the differences between either the wetter and dryer scenarios significantly different from the average scenario. A significant difference in outcomes (let's say of $5 \%$ or more) of either a wetter or dryer, might suggest that policy makers should display some caution before committing to an adaptation response, because the uncertainty of the investment returns would be greater than the rate of interest associated with obtaining the funds. Second, are the differences in impacts of a dryer scenario away from the average similar to those from a wetter scenario away from the average. If this is the case, then again some caution should be exercised by the policy maker, because it implies that the impacts are not going to be the same across the catchment. This occurs because the water resource systems are configured to work under a select set of parameters, only one of which is the rate of precipitation. So it could be the case that even though it is wetter, the system is such that it cannot use the extra water, thus evoking development of it. Conversely, this investment would be wasted if it turns out to be dryer.

\section{Aims}

This paper addresses an approach to the problem of not knowing how to adapt to climate change because there is uncertainty in what that climate outcome might be. The issues are discussed in terms of a case study taken from the Musi Catchment of the Krishna River basin in India. Of interest are the physical and economic impacts of a range of different climate scenarios on a complex catchment which has different end-uses of water (urban, industrial, irrigation and dryland agriculture), spread across a diverse region in which both surface and groundwater are accessed. Because of its diversity, the Musi catchment can be representative of many other catchments, even though others may differ in terms of size and scope.

The aim in this paper is to evaluate the physical and economic impact of climate change on the Musi catchment over the immediate future (2011 to 2040), in order to ascertain the differences between an average climate, a dry climate and a wet climate. If the differences are great, then it is presumed that policy makers should exercise some caution before committing resources to adapt to climate change as they may well be wasted.

\section{Conditions that need to be considered}

In order to analyse this problem, three important facts need to be considered. First, that the impact of climate change will be complex and affect different parts of the catchment in different ways, depending on how the water supply is affected and how the water is utilised. Second, that the way the system is run will have a bearing on the physical and economic outcomes from the system. Third, that it is unknown whether the future will be dryer or wetter.

The first of these facts can be handled by using a hydro-economic simulation model that accounts for the different demands and supplies of water across a catchment. Not only do the physical elements of supply and demand need to be lined up in one contiguous unit, but some recognition needs to be made regarding the value of water changing across the catchment according to use.

The second problem of calculating how it is run can be accommodated by observing the reliability with which water is provided to different parts of the system. So, a very conservative operation strategy might be one where water is guaranteed 90 years in 100, a less conservative one would be less. In this study the range of 70, 80 and $90 \%$ reliability is imposed on the system.

Finally, the hydro-economic simulation model needs to be informed by different climate scenarios. In this analysis, it is necessary to assess the impacts that arise from changes/differences in the climate only. Three climate scenarios can be considered; one that is an average middle course, another that would be wetter than expected and a third that is dryer.

\section{What needs to be compared?}

Overall there is a need to know what happens into the future, first if the system does continue on some medium arbitrary path, (known as Qave). From this base there is a need to know what happens to the flows of water and income that arise if the climate is wetter (Q0) or dryer (Q1) from this average path. So, of interest in this paper is the absolute and relative differences the wetter and dryer climate scenarios have from the average (Qave), not what the actual absolute levels are.

The key performance variables of interest are the differences that arise between average and extreme climate scenarios on the: 
- Quantity of water reliably supplied to each node each year, measured in terms of MCM per annum, but shown as a percentage change.

- Net benefits (total returns minus total costs) derived from the water reliably supplied to each node, specified as an annual average earned over the 19-year period modelled, measured in terms of Rs.million, but revealed as a percentage change.

\section{Methods and assumptions}

The analysis was conducted on the Musi catchment in India (see Figure 1). The catchment is subdivided into four dryland zones, urban use in Hyderabad, Industrial uses and some irrigation in the wastewater region, the Anicutt and the medium Musi scheme. There are water imports from outside the catchment from the Nagarjuna Sagar system that while they are considered in the overall system modelling, they are excluded from this analysis. Details of the model are presented in Davidson et al. (forthcoming). This model is fed by a climate model that is used to assess groundwater and surface flow resources. Allocations to the major urban centre of Hyderabad, the industrial sector, four separate irrigation zones and four dryland zones are included as demand centres. From this model, a set of flow duration curves are produced for each node, thus providing information on the quantity of water that can be supplied to each node at different reliability levels.

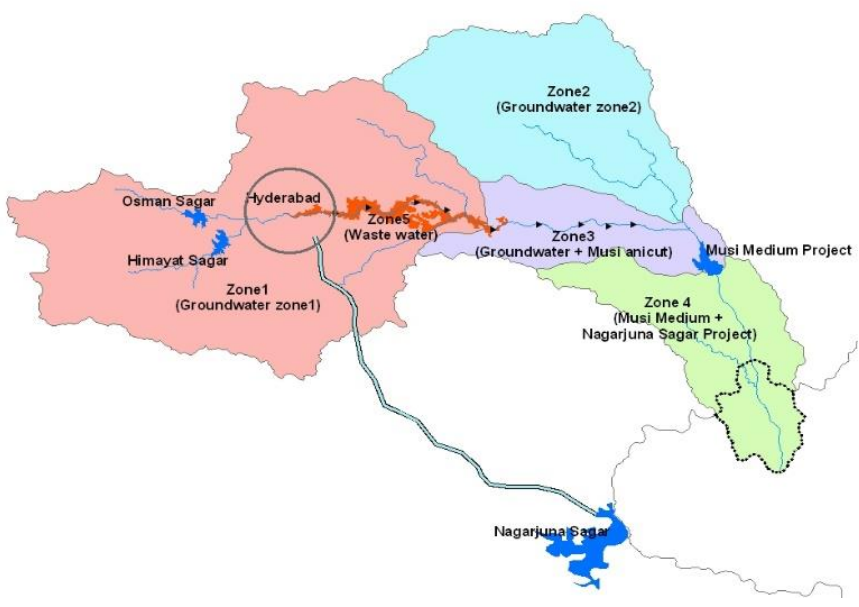

Fig. 1: The Musi Catchment.

Future climate variants were generated by downscaling climate predictions carried out using the Global Climate Model HADGEM. PRECIS generated three QUMP (Quantifying Uncertainty in Model Predictions) simulation outputs $\left(\mathrm{Q}_{0}, \mathrm{Q}_{1}, \mathrm{Q}_{14}\right)$ under the IPCC A1B scenarios corresponding to the baseline (1980-2010) and the early-century (2011-2040) extracted by Indian Institute of Tropical Meteorology (IITM) have been used in this study. While the mid-century (20412070) and end-century (2071-2098) exist, they are not employed in this study. The downscaled climate data is used as forcing data in the hydrologic and groundwater model and simulations are carried out using the climate projection data. For further results and details on the climate models see Malano et al, (2015).

The SWAT model (Arnold et al. 2005), a semi-distributed model surface hydrology model in combination with the groundwater model MODFLOW are applied to assess the overall impacts of each climate scenario on the hydrologic behaviour of the catchment, and provide a quantified assessment of water resources availability under these climates.

Water security is the product of combining water resources availability (inflows, groundwater, recycled water, return flows) and water demands (irrigation, urban, industry and environmental) imposed on the resource across the whole of the catchment taking into consideration all the water uses and related infrastructure. The water security assessment is carried out by combining these two domains using the Water Resource Allocation Model, REALM (George et al. 2011a, 2011b). 
The allocation model, REALM was then applied to assess the water resources available to various demand centres based on user-defined demand priorities and preferences. The REALM model generates several outputs including unmet demand (shortfall) and volume supplied for each demand node for each month of the simulated time series, which are then aggregated to calculate the annual water supply. These values are then used to determine the reliability of supply employing frequency analysis.

The allocation model was first calibrated and validated before using it to simulate the performance of adaptation responses like cropping pattern change, increased irrigation efficiency, and increased watershed development. To evaluate the hydrologic performance of the adaptation responses, the allocated volume of water to each demand centre under these scenarios is compared with the business-as-usual scenario (BAU).

The allocation model is intended to simulate future adaptation responses to climate change in the Musi sub-basin. In this paper, the REALM model is applied to a Business-As-Usual (BAU) scenario, which is based on the expected future demand from each sector (urban, industry and agriculture) together with river streamflow and groundwater recharge estimates under the ensemble average climate scenario. The ensemble climate series was generated by averaging three QUMP variants $\left(\mathrm{Q}_{0}, \mathrm{Q}_{1}, \mathrm{Q}_{14}\right)$. This climate series was used as forcing variables in the SWAT and MODFLOW models to generate stream flows and groundwater recharge.

From an economic perspective, these quantities of water reliably supplied are valued at the average rate at which water is valued at each node, as calculated by Hellegers and Davidson (2011). It should be noted that according to Davidson et al.(forthcoming) supplying water more reliably comes at a cost, especially of that water that could have been used if it were not held in reserve to maintain a higher level of reliability. That approach to valuation is used in this paper.

In particular, it should be noted that in this analysis it is assumed that:

- No modifications or adaptations are made to the system in light of climate change

- The value of water does not change over the period of analysis.

- That while different nodes and levels of reliability are observed and commented on, the only factor causing changes in this model are different forcing's from the climate component of the model.

\section{Results and implications}

The results of the analysis are presented in Table 1. It should be noted depending on the way the system is run (either with 70,80 or $90 \%$ reliability levels) affects the results greatly. As was expected, as the system is run more reliably the impacts become more pronounced. Trying to run the system at a 90\% reliability level appears to double the impacts over what occurs at the $70 \%$ level. This implies that policy makers have some flexibility in the way they run their systems and that running them at different levels of reliability may be an ideal climate change adaptation strategy.

To simplify and illustrate the impacts a wetter or dryer outcome may have on the system, the results for running the system at the 70\% reliability level are presented in Figures 2 and 3. Immediately of interest are that the direction of the physical and economic impacts is the same, something that is not surprising given that the economic impacts are derived from the physical flows. However, the magnitude between the two is very different, with in general the economic impacts being greater in percentage difference terms than the physical impacts. This result implies that policy makers will need to consider the economic consequences of any adaptation strategy and not confine the decisions to the purely physical.

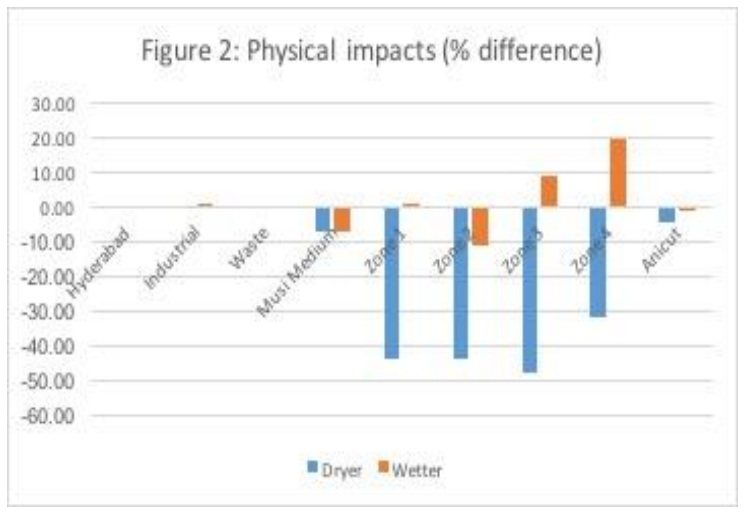

Fig. 2: Physical impacts (\% difference). 


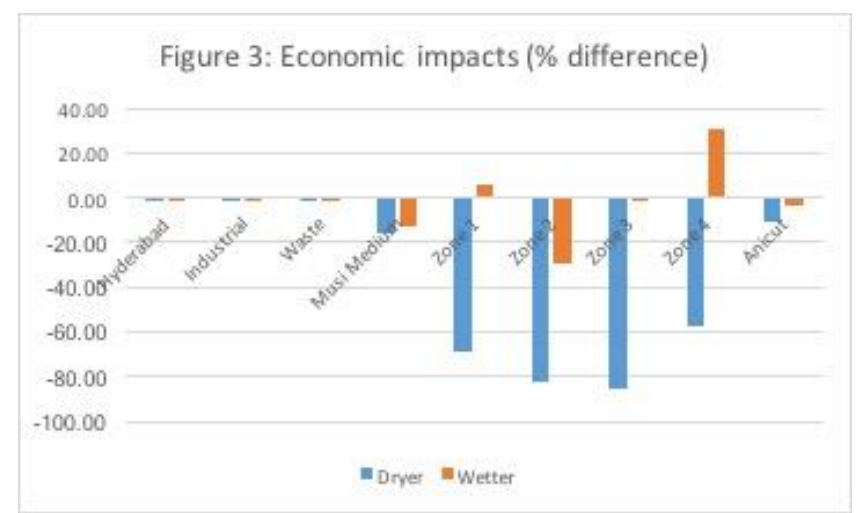

Fig. 3: Economic impacts (\% difference).

It should be noted that regardless of the scenario chosen, the impacts on Hyderabad City and the industrial sector are very small. The changes in allocations are all less than $1 \%$, regardless of how reliable the system is run. This change has a concomitant flow on effect down the Musi, through the Wastewater region (which derives most of its supplies as effluent from Hyderabad city). The impacts are somewhat greater the further one travels down the Musi, with the Musi Medium experiencing a change in the order of $10 \%$ (7\% when run at $70 \%$ reliability and $13 \%$ when run at $90 \%$ reliability) in water allocated and the Anicut a little less. Thus any adaptation policy directed towards these regions will not be affected by differences in the climate outcomes. Concerns about a wetter or dryer scenario should not figure greatly in decisions directed towards climate change adaptation.

The largest impacts in differences away from what might be expected on average occur in the dryland agricultural zones. While the quantities are between 30 and up to $70 \%$ away from the average. Furthermore, in general, the changes that occur when the system is dryer are greater than if it is wetter, by a factor of four. This would imply that the down side risks associated with climate change are greater that the upside risks.

From an economic perspective changes in climate were estimated to have the greatest impact on Zone 1, a dryland region close to Hyderabad. This occurs because the value of production in this region is higher, principally centred vegetable and fruit crops. The losses from a dryer climate would appear to be far greater than the gains if the climate turns out to be wetter. Once again, this implies that the downside risks of future climate change are greater than the upside risks. Thus, A dryer climate will have a greater impact than a wetter one. In Hyderabad City, the industrial and wastewater zone, the changes in economic impacts is relatively small. This reflects the physical changes that are observed above. However, in the dry zones the percentage change in net benefits is extremely high. The impacts, both from wetter and dryer outcomes will affect farmers greatly. 
Table 1: Results.

\begin{tabular}{|c|c|c|c|c|c|c|c|c|c|c|}
\hline Q1-Qave & Reliability ( & lerabad & strial & Waste & Musi Medit & Zone 1 & Zone 2 & Zone 3 & Zone 4 & Anicut \\
\hline \multirow[t]{3}{*}{ (dryer) } & 70 & -3.29 & -0.34 & -0.37 & -7.59 & -195.04 & -85.35 & -49.36 & -52.96 & -2.8 \\
\hline & 80 & -3.25 & -0.25 & -0.52 & -9.61 & -206.59 & -91.28 & -54.01 & -59.14 & -3.98 \\
\hline & 90 & -3.18 & -0.12 & -0.72 & -12.4 & -222.62 & -99.53 & -60.46 & -67.71 & -5.63 \\
\hline Q0-Qave & 70 & -1.11 & 0.47 & -0.13 & -7.35 & 0.93 & -21.85 & 8.84 & 32.61 & -0.70 \\
\hline \multirow[t]{2}{*}{ (wetter) } & 80 & -2.31 & 0.31 & -0.29 & -8.97 & 2.04 & -28.12 & 7.81 & 29.45 & -1.45 \\
\hline & 90 & -4.41 & 0.06 & -0.60 & -11.22 & -0.28 & -37.53 & 7.65 & 20.90 & -2.49 \\
\hline \multicolumn{11}{|c|}{ Precentage change from Qave } \\
\hline \multirow{3}{*}{$\begin{array}{l}\text { ( Q1-Qave) } \\
\text { (dryer) }\end{array}$} & 70 & -0.56 & -0.28 & -0.25 & -7.39 & -43.86 & -43.70 & -47.82 & -31.95 & -4.52 \\
\hline & 80 & -0.60 & -0.23 & -0.35 & -9.69 & -49.45 & -51.24 & -56.37 & -38.67 & -6.86 \\
\hline & 90 & -0.67 & -0.12 & -0.48 & -13.17 & -58.52 & -64.47 & -70.67 & -50.10 & -10.70 \\
\hline \multirow{3}{*}{$\begin{array}{l}\text { (Q0 - Qave } \\
\text { (wetter) }\end{array}$} & 70 & -0.19 & 0.39 & -0.09 & -7.16 & 0.21 & -11.19 & 8.56 & 19.67 & -1.13 \\
\hline & 80 & -0.43 & 0.28 & -0.19 & -9.05 & 0.49 & -15.78 & 8.15 & 19.25 & -2.50 \\
\hline & 90 & -0.93 & 0.06 & -0.40 & -11.91 & -0.07 & -24.31 & 8.94 & 15.46 & -4.73 \\
\hline & & & & & & & & & & \\
\hline \multicolumn{11}{|c|}{ Economic (Rs.million average annual benefit) } \\
\hline \multirow{3}{*}{$\begin{array}{l}\text { Q1-Qave } \\
\text { (dryer) }\end{array}$} & 70 & -140 & -8 & -2 & -35 & -1277 & -238 & -130 & -133 & -13 \\
\hline & 80 & -155 & -6 & -3 & -41 & -1367 & -255 & -141 & -152 & -16 \\
\hline & 90 & -227 & -3 & -4 & -55 & -1478 & -278 & -156 & -180 & -22 \\
\hline \multirow{3}{*}{$\begin{array}{l}\text { Q0-Qave } \\
\text { (wetter) }\end{array}$} & 70 & -286 & -5 & -1 & -28 & 112 & -86 & -1 & 71 & -4 \\
\hline & 80 & -435 & -8 & -2 & -32 & 94 & -96 & 0 & 64 & -4 \\
\hline & 90 & -774 & -18 & -3 & -41 & 78 & -128 & 1 & 33 & -9 \\
\hline \multicolumn{11}{|c|}{ Percentage change from Qave. } \\
\hline ( Q1-Qave) & 70 & -0.40 & -0.58 & -0.65 & -15.77 & -68.48 & -82.35 & -85.67 & -57.85 & -10.43 \\
\hline \multirow[t]{2}{*}{ (dryer) } & 80 & -0.51 & -0.53 & -0.85 & -19.94 & -80.97 & -103.92 & -106.44 & -75.51 & -14.64 \\
\hline & 90 & -1.01 & -0.35 & -1.12 & -29.07 & -107.26 & -164.88 & -153.89 & -120.61 & -25.16 \\
\hline \multirow{3}{*}{$\begin{array}{l}\text { (Q0 - Qave } \\
\text { (wetter) }\end{array}$} & 70 & -0.82 & -0.35 & -0.34 & -12.67 & 6.00 & -29.74 & -0.74 & 31.02 & -3.35 \\
\hline & 80 & -1.44 & -0.65 & -0.48 & -15.18 & 5.58 & -39.27 & -0.21 & 31.67 & -3.72 \\
\hline & 90 & -3.42 & -1.89 & -0.81 & -21.66 & 5.66 & -76.01 & 1.07 & 22.14 & -10.55 \\
\hline
\end{tabular}

\section{Discussion and Implications}

The implications from this research are quite profound. When analysing climate change a range of outcomes is possible, some which might result in a wetter than expected outcome or a dryer outcome. It would appear from both an economic and physical perspective, that policy makers should be worried about a dryer outcome. The risks to the agricultural sector, in particular, are quite extreme if the climate turns out to be dryer.

It would appear that climate change will have a different impact across the catchment. While those in the urban sector and in the industrial sector remain somewhat unaffected by it due to the high priority of supply, those in the rural sector will bear much of the burden of what is occurring. Physically it would appear that the dryland zones are not as greatly affected, however from an economic perspective the impact will be significant. However, what should not be forgotten is that while the percentage change in economic benefits in Hyderabad City is not great, it still represents a significant loss during a dryer climate of between Rs.286 million and Rs.774million, depending on how reliably the system is operated. Policy makers should concentrate on mitigating the potential losses from Dryland zone 1, the place where the highest value crops are grown.

The impacts that arise from the way the system is run are of a similar magnitude to those that arise from changes in the climate. In other words, running the system less reliably can be used to mitigate the effects of climate. 


\section{Conclusions}

Policy makers need a strategy to handle the uncertainty that comes from different views about what a climate is likely to be, before they commit resources that are dedicated to adapting to climate change. The strategy suggested in this paper is one in which wetter and dryer outcomes derived from a climate model are compared to an average outcome. When a coupled hydro-economic model is used to simulate the different climate forcings, the physical and economic impacts can be clearly observed. In places where the differences are not great, any policy directed to the problem is unlikely to be affected by an unexpected difference in the climate. In addition, it is possible to identify where some caution needs to be exercised.

\section{References}

[1] J. G. Arnold, F. Nancy, "SWAT2000: Current capabilities and research opportunities in applied watershed modelling," Hydrological Processes, vol. 19, no. 3, pp. 563-572, 2005. 